training, specific care processes, EMR prompts for tests and treatments, regularly reviewed process metrics and group financial incentives. Practice variance was reduced and outcomes markedly improved.

Implications for Guideline Developers Guideline recommendations are more likely to be adopted in a uniform manner if they include specific recommendation, suggestions for implementation use in organised settings, and process and outcome metrics to track improvements.

\section{BEST PRACTICES AND PERFORMANCE MEASURES FOR SYSTEMIC TREATMENT PERSCRIBER ORDER ENTRY SYSTEMS (STCPOE) IN CHEMOTHERAPY DELIVERY}

V Kukreti, A Cheung, S Hertz, L Kaizer, S Lankshear. Cancer Care Ontario, Toronto, Canada

\section{0:1136/bmjgs-2013-002293.51}

Background While information technology (IT) has the potential to improve the quality and safety of patient care, solutions such as computerised physician order entry (CPOE) are often designed and executed without end-user involvement and lack performance measures for monitoring quality and impact. To address this gap, an evidence based guideline for systemic treatment (ST) CPOE was developed incorporating both clinical and technological best practices. Performance measures for monitoring clinical impacts and system functionality were also developed.

Context The ST CPOE guideline was developed by a panel of physicians, nurses, pharmacists, IT specialists and human factors experts. Two Expert Panels (i.e. Clinical and Technology) were convened, to review and provide feedback on guideline content. Description of Best Practice The guideline contains two distinct yet interconnected parts: clinical practice (e.g. error prevention, utilisation, clinical decision support), and technology requirements (e.g. usability, system integration, effective alerts). Also included are evidence based indicators to support the evaluation of ST CPOE systems and indicators reflecting clinician practice and patient outcomes. Quality monitoring of ST CPOE utilisation reveal that $75.5 \%$ of all chemotherapy visits are being supported by an ST CPOE system. A provincial evaluation of existing ST CPOE systems against the technology best practices is currently underway.

Lessons for Guideline Developers, Adaptors, Implementers, and/or Users This innovative guideline focuses on clinical practice driving IT solutions, not the other way around. A priori commitment to indicator development allowed for expanding beyond describing best practices to including indicators for monitoring progress toward achieving best practice, thus increasing relevance and uptake by end users.

\section{REDUCING OVERPOPULATION: ACHIEVING MORE BY DOING LESS}

J Schottinger, M Koster. Kaiser Permanente, Southern California Permanente Medical Group, Pasadena, USA

\section{0:1136/bmjqs-2013-002293.52}

Background Too-frequent screening for cervical cancer can increase costs, lead to unnecessary invasive procedures associated with overtreatment, and shift resources away from the one in five women who do not receive recommended routine screening.
Context A large, US-based integrated healthcare system with centralised evidence services and eight independent regions developed and implemented an evidence-based guideline for cervical cancer screening. Novel implementation strategies and performance monitoring in one region in Southern California led to significant improvements and are described below.

Description of Best Practice Graded evidence summaries were conducted by a centralised analytic unit, and recommendations developed by a guideline team with representation from each region. In one large region with more than 3 million patients, interventions aimed at the practitioner, patient and systems levels were implemented for routine Pap and HPV co-testing. Practitioner interventions included electronic distribution of guidelines, point-of-care electronic prompts, and workflow support. Patient-level interventions included point-of-care education, and in-reach/outreach activities. System-level interventions focused on centralised patient outreach letters and reminder calls, computerised decision support, and unscreened cancer lists for panel management. Monthly performance monitoring on a measure of "overpopulation" was reported at medical centre, department and provider levels. In a five-year period, over 100,000 fewer unnecessary Pap tests were performed, while screening rates increased by $7 \%$.

Lessons for Guideline Developers, Adaptors, Implementers, and/or Users Centralised guideline development, coupled with coordinated implementation and performance monitoring, can reduce unnecessary screening and invasive procedures, focus resources on appropriate routine screening in underscreened populations, improve patient access and reduce costs.

\section{DEVELOPING GUIDELINES AND QUALITY INDICATORS SIMULTANEOUSLY: EFFECTS ON GUIDELINE CONTENT AND IMPLICATIONS ON THE GUIDELINE DEVELOPMENT PROCESS}

${ }^{1} \mathrm{M}$ Follmann, ${ }^{1} \mathrm{~S}$ Wesselmann, ${ }^{2} \mathrm{~K}$ Kopp, ${ }^{2} \mathrm{M}$ Nothacker. 'German Cancer Society, Berlin, Germany; ${ }^{2}$ Association of the Scientific Medical Societies, Duesseldorf, Germany

\section{0:1136/bmjqs-2013-002293.53}

Background The German Guideline programme in Oncology (GGPO) funds and supports the development, implementation and evaluation of evidence based guidelines. An essential part of the programme is the development of quality indicators (QI) before a guideline is published. QI groups representing the multidisciplinary guideline development group including patient representatives and experts from organisations responsible for QI assessment and evaluation realise this following a standardised methodology.

Objectives To explore the effects of a standardised Quality Indicator Development Process (QIDP) on the content of guidelines and possible implications on the guideline development process. Methods Retrospective content analysis of current guideline manuscripts. Description and categorization of changes in the guideline draft after the QIDP. Structured interview of QI groups.

Results 9 oncological guidelines including 87 QI were analysed. Changes in guideline drafts after the QIDP included: - formulation of new recommendations - specification of the wording of recommendations - specification and amendment of the predefined aims of a guideline - identification of aspects to consider for an update of the guideline. Results of the interview will be presented at the conference. 